\title{
Importance sampling the Rayleigh phase function
}

\section{Frisvad, Jeppe Revall}

Published in:

Optical Society of America. Journal A: Optics, Image Science, and Vision

Link to article, DOI:

10.1364/JOSAA.28.002436

Publication date:

2011

\section{Document Version}

Publisher's PDF, also known as Version of record

Link back to DTU Orbit

Citation (APA):

Frisvad, J. R. (2011). Importance sampling the Rayleigh phase function. Optical Society of America. Journal A: Optics, Image Science, and Vision, 28(12), 2436-2441. https://doi.org/10.1364/JOSAA.28.002436

\section{General rights}

Copyright and moral rights for the publications made accessible in the public portal are retained by the authors and/or other copyright owners and it is a condition of accessing publications that users recognise and abide by the legal requirements associated with these rights.

- Users may download and print one copy of any publication from the public portal for the purpose of private study or research.

- You may not further distribute the material or use it for any profit-making activity or commercial gain

- You may freely distribute the URL identifying the publication in the public portal

If you believe that this document breaches copyright please contact us providing details, and we will remove access to the work immediately and investigate your claim 


\title{
Importance sampling the Rayleigh phase function
}

\author{
Jeppe Revall Frisvad \\ Department of Informatics and Mathematical Modelling, Technical University of Denmark, Asmussens Alle, \\ Building 305, DK-2800 Kgs. Lyngby, Denmark (jrf@imm.dtu.dk)
}

Received August 4, 2011; revised September 23, 2011; accepted September 23, 2011; posted September 26, 2011 (Doc. ID 152330); published November 10, 2011

\begin{abstract}
Rayleigh scattering is used frequently in Monte Carlo simulation of multiple scattering. The Rayleigh phase function is quite simple, and one might expect that it should be simple to importance sample it efficiently. However, there seems to be no one good way of sampling it in the literature. This paper provides the details of several different techniques for importance sampling the Rayleigh phase function, and it includes a comparison of their performance as well as hints toward efficient implementation. (c) 2011 Optical Society of America OCIS codes: $\quad 290.5870,290.4210,010.5620,000.5490$.
\end{abstract}

\section{INTRODUCTION}

Rayleigh scattering is a broadly applied model for the scattering of spherical particles smaller than the wavelength of the incident light. The textbook use of Rayleigh scattering is to explain the color of the sky [1]. Molecular scattering in both atmosphere and ocean is the classical application of the model [2-4]. Rayleigh scattering also has other uses, including scattering in tissue [5-9], astrophysical radiative transfer $[\underline{10}, \underline{11}]$, and nanoporous materials [12]. These application areas all involve, in one way or another, Monte Carlo simulation for computing multiple scattering effects. So there is no doubt that the Rayleigh phase function has been sampled in many different simulation programs and in many different ways. I am, however, unable to find a detailed description in the literature of how to importance sample it correctly. This paper provides the details of several different techniques for importance sampling the Rayleigh phase function, and it includes a comparison of their performance as well as hints toward efficient implementation. Such an investigation should be useful in the diversity of research fields that involve Monte Carlo simulation of light scattering. More specifically, it is useful in Monte Carlo simulations that solve any light transport or radiative transfer problem involving scattering by particles smaller than the wavelength of the incident illumination.

One might argue that the Henyey-Greenstein phase function [13] is the more common choice, so why bother looking at the Rayleigh phase function? The Rayleigh phase function is still important because it is more correct than the HenyeyGreenstein phase function in describing the scattering from very small particles $[\underline{10}, \underline{14}, \underline{15}]$. The asymmetry parameter $g$, which is the mean cosine of the scattering angle, is zero for very small particles. In this case, the Henyey-Greenstein phase function becomes the isotropic phase function, which does not capture the angular dependency of the scattering as the Rayleigh phase function does.

\section{RAYLEIGH SCATTERING}

Scattering by spherical particles of a size smaller than the wavelength of the incident light was investigated by John William Strutt (later Lord Rayleigh) in the second half of the nineteenth century [16]. The scattering exhibited by such small particles is today referred to as Rayleigh scattering. The following is Lord Rayleigh's verbal formulation of his results [16]:

When light is scattered by particles which are very small compared with any of the wavelengths, the ratio of the amplitudes of the vibrations of the scattered and incident light varies as the square of the wavelength and the intensity of the lights themselves as the inverse fourth power.

The mathematical formulation of Lord Rayleigh was a formula that returns the scattered light as a function of wavelength $\lambda$, distance to scattering event $r$, scattering angle $\theta$, particle and host medium density, number of particles $N$, and particle volume $T=\frac{4}{3} \pi a^{3}$, where $a$ is the particle radius. The modern formulation of Rayleigh scattering starts with scattering properties, works with the particle refractive index $n$ (rather than densities), and includes particle polarizability $\alpha$. The result, however, differs only by a factor from Lord Rayleigh's original result.

Starting from scattering properties, the Rayleigh phase function is ([17], Section 3)

$$
p_{R}(\cos \theta)=\frac{1}{4 \pi} \frac{3}{4}\left(1+\cos ^{2} \theta\right),
$$

where the normalizing division by $4 \pi$ has been included. The scattering cross section of a spherical particle small compared to the wavelength is ([1] ], Section 16)

$$
C_{s}=\frac{128 \pi^{5}}{3 \lambda^{4}} \alpha^{2}
$$

Using the Clausius-Mossotti (or Lorentz-Lorenz) equation, a spherical particle in air has polarizability

$$
\alpha=\frac{n^{2}-1}{n^{2}+2} a^{3} .
$$

Now, suppose we have a light ray of radiance $L_{i}$ in an element of solid angle $d \omega^{\prime}$ of direction $\vec{\omega}^{\prime}$ and wavelength $\lambda$ 
incident on a medium with a number density $N$ of small particles, then the radiance scattered in the direction $\vec{\omega}$ is

$$
\sigma_{s} p_{\mathrm{R}}\left(\vec{\omega} \cdot \vec{\omega}^{\prime}\right) L_{i} d \omega
$$

where $\sigma_{s}$ is the scattering coefficient. We can compute the scattering coefficient from the number density of particles:

$$
\sigma_{s}=\int_{0}^{\infty} N(a) C_{s}(a) \mathrm{d} a
$$

If all the particles are of the same radius $a$, we have

$$
\sigma_{s}=N C_{s}
$$

The scattered ray originates in a point and spreads in a solid angle $d \omega$ around the direction $\vec{\omega}$. Once the ray reaches a surface, the element of solid angle is

$$
d \omega=\frac{d A_{s}}{r^{2}}
$$

where $d A_{s}$ is the element of spherical surface area on the unit sphere subtended by $d \omega$ and $r$ is the distance from the point of scattering to the surface reached by the ray. This is the reason why attenuation by the squared distance to the scattering event $r^{2}$ is sometimes included instead of the element of solid angle $d \omega$ in the formula for Rayleigh scattering.

If we use the information provided above, we get the following formula for Rayleigh scattering:

$$
L_{s}=N \frac{8 \pi^{4} a^{6}}{\lambda^{4}}\left(\frac{n^{2}-1}{n^{2}+2}\right)^{2}\left(1+\left(\vec{\omega} \cdot \vec{\omega}^{\prime}\right)^{2}\right) L_{i} d \omega,
$$

where $L_{i}$ is incident radiance from the direction $\vec{\omega}^{\prime}$ and $L_{s}$ is scattered radiance in the direction $\vec{\omega}$, while $N$ is the number density of small spherical particles of radius $a$ and refractive index $n$ illuminated by light of wavelength $\lambda$. Integrating over all incoming directions, this is the source (or in-scattering) term of the radiative transfer equation as it would appear for turbid materials or participating media that exhibit Rayleigh scattering. In order to evaluate the radiative transfer equation for such materials or media efficiently using Monte Carlo integration, we need a way of importance sampling the Rayleigh phase function $p_{R}$.

\section{SAMPLING THE RAYLEIGH PHASE FUNCTION}

In the following, we let $\xi$ and $\xi_{i} \in[0,1], i=1,2, \ldots$, denote uniform, random variables. For starters, we sample the unit sphere uniformly (the isotropic phase function) and see how well the samples are distributed over the shape of the Rayleigh phase function. There are two well-known ways to sample a direction on the unit sphere. We can use the von Neumann rejection technique [18], which is simply do

$(x, y, z)=\left(2 \xi_{1}-1,2 \xi_{2}-1,2 \xi_{3}-1\right)$

while

$x^{2}+y^{2}+z^{2}>1$,

$\vec{\omega}=(x, y, z) /\left(x^{2}+y^{2}+z^{2}\right)^{1 / 2}$,

or we can sample the direction in spherical coordinates by

$$
(\theta, \phi)=\left(\cos ^{-1}\left(2 \xi_{1}-1\right), 2 \pi \xi_{2}\right),
$$

where we, of course, do not compute $\theta$ but use $\cos \theta=2 \xi_{1}-1$ directly. According to Kalos and Whitlock ([19], p. 72), the efficiency of the two methods is comparable in two dimensions. This is not necessarily true in three dimensions as the bound is not as tight in the rejection sampling. Let us refer to the function that implements either of these two methods as sample_isotropic(). A uniform sampling of the unit sphere (i.e., no importance sampling) would sample the Rayleigh phase function as shown in Fig. 1(a). The samples are clustering in the middle area where the contributions of the rays are smaller. This is suboptimal.

One way to importance sample the Rayleigh phase function is using (more) rejection sampling. This is done by finding a probability density function $h$, which we know how to sample and which, multiplied by a constant $c$, is always greater than the function $f$, which we would really like to sample. The condition for acceptance of a sample is then ([20], Subsection 14.3.5)

$$
\xi<\frac{f(x)}{\operatorname{ch}(x)}
$$

Considering $p_{R}$, the probability density function (pdf) that we would like to sample, we can see that

$$
\max p_{\mathrm{R}}=\frac{1}{4 \pi} \frac{3}{4}\left(1+1^{2}\right)=\frac{3}{2} \frac{1}{4 \pi},
$$

and uniform sampling of a direction on the unit sphere has pdf $=\frac{1}{4 \pi}$. The constant would then be $c=\frac{3}{2}$. Then the rejection sampling is as follows:

$$
\begin{aligned}
& \text { do } \\
& \vec{\omega}=\text { sample_isotropic }() \\
& \text { while } \xi>\frac{1}{2}\left(1+\left(\vec{\omega} \cdot \vec{\omega}^{\prime}\right)^{2}\right),
\end{aligned}
$$

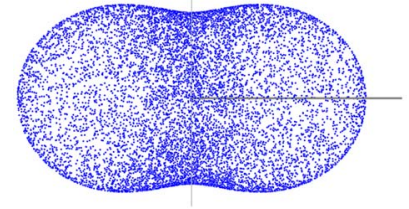

(a)

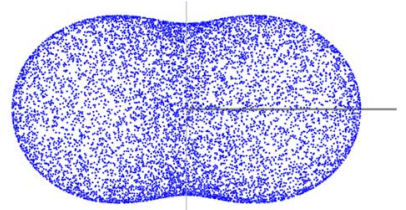

(b)
Fig. 1. (Color online) Spread of direction samples as points on the shape of the Rayleigh phase function. Directions sampled (a) uniformly across the unit sphere and (b) using rejection techniques. 
where $\vec{\omega}^{\prime}$ is the forward direction. Figure $\underline{1(b)}$ illustrates how this sampling scheme distributes the samples uniformly over the shape of the Rayleigh phase function.

\section{A. Simplified Rejection Sampling around the Forward Direction}

If we sample the direction in spherical coordinates $(\theta, \phi)$ with the forward direction $\vec{\omega}^{\prime}$ as the zenith direction, we can simplify the algorithm somewhat:

$$
\begin{aligned}
& \text { do } \\
& \cos \theta=2 \xi_{1}-1 \\
& \text { while } \xi_{2}>\frac{1}{2}\left(1+\cos ^{2} \theta\right), \\
& \phi=2 \pi \xi_{3} .
\end{aligned}
$$

We can translate these spherical coordinates to a Cartesian unit vector by

$$
{ }_{\perp} \vec{\omega}=(x, y, z)=(\sin \theta \cos \phi, \sin \theta \sin \phi, \cos \theta),
$$

where the subscript $\perp$ signals that these are coordinates with respect to a basis $\left\{\vec{b}_{1}, \vec{b}_{2}, \vec{\omega}^{\prime}\right\}$, where $\vec{\omega}^{\prime}$ is the direction of the $z$ axis. We have no explicit knowledge about the other two vectors of this basis. Therefore, we must build an orthonormal basis from $\vec{\omega}^{\prime}$ in order to find the coordinates of $\vec{\omega}$ in the usual basis. Once the two other basis vectors have been chosen, the change of basis is

$$
\vec{\omega}=x \vec{b}_{1}+y \vec{b}_{2}+z \vec{\omega}^{\prime}
$$

There are several ways to build the vectors $\vec{b}_{1}$ and $\vec{b}_{2}$ from $\vec{\omega}^{\prime}$. The usual approach is to find a vector orthogonal to $\vec{\omega}^{\prime}$, normalize it, and take the cross product of this vector and $\vec{\omega}^{\prime}$ to find the third vector in the basis. See for example the method of Hughes and Möller [21]. This transformation from spherical coordinates to a Cartesian unit vector in the usual basis carries some overhead, as compared to isotropic sampling, since it involves the sines and cosines of $\theta$ and $\phi$ as well as the change of basis. Note, however, that $\cos \theta$ is given directly and we can get $\sin \theta$ by

$$
\sin \theta=\left(1-\cos ^{2} \theta\right)^{1 / 2}, \quad \text { since } \theta \in[0, \pi] .
$$

A similar rejection sampling technique that uses $\cos \theta=$ $\cos \left(\pi \xi_{1}\right)$ has been proposed by Baranoski and coworkers [6-9]. This approach requires a correction of the acceptance condition in the rejection sampling to return the right result. The correction is missing in the mentioned references.

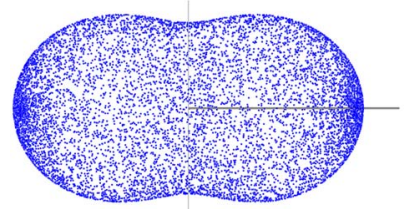

(a)

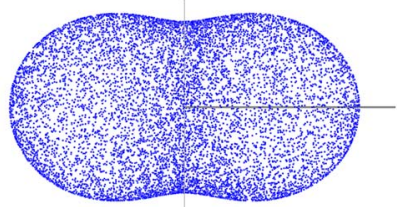

(b)
Fig. 2. (Color online) Spread of direction samples as points on the shape of the Rayleigh phase function. Directions sampled using Baranoski's rejection sampling (a) without correction and (b) with correction.
Figure 2(a) illustrates how the samples cluster around the forward and backward directions if we do not make the correction. By going through the steps of the inversion method ([19], pp. 45-48) in reverse order, we can find out what pdf $\cos \left(\overline{\pi \xi_{1}}\right)$ is really sampling. We have that the complementary cumulative distribution function is

$$
\operatorname{ccdf}(\cos \theta)=\frac{1}{\pi} \cos ^{-1}(\cos \theta) .
$$

This means that

$$
\operatorname{pdf}(\cos \theta)=\frac{1}{\pi}\left(1-\cos ^{2} \theta\right)^{-1 / 2},
$$

and the constant that makes it the tightest possible bound of $p_{R}$ is $\sqrt{6} / 12$. Computing this constant is slightly involved. First, we equate the original pdf with the other pdf multiplied by a factor $c$ :

$$
\begin{aligned}
\frac{1}{4 \pi} \frac{3}{4}\left(1+\cos ^{2} \theta\right) & =\frac{c}{\pi}\left(1-\cos ^{2} \theta\right)^{-1 / 2} \\
& \Rightarrow \underset{\sin \theta>0}{\Rightarrow} c=\frac{3}{16} \sin \theta\left(1+\cos ^{2} \theta\right) .
\end{aligned}
$$

With this equation, we can find the constants that ensure that the two pdfs intersect at some point. The two curves can have two, three, or four intersection points. What we want is two intersection points, otherwise the constant times the pdf is not always larger than $p_{R}$; see Fig. 3. This happens where the equation for $c$ finds its maximum (take the derivative of the equation for $c$ with respect to $\theta$ and set it equal to 0 ). The maximum is at $\cos \theta=\sqrt{3} / 3$. Inserting this to find $c$, we get $c=\sqrt{6} / 12$. Thus, this constant gives the tightest possible bound. The right way to use Baranoski's rejection sampling is then

$$
\begin{aligned}
& \text { do } \\
& \cos \theta=\cos \left(\pi \xi_{1}\right) \\
& \text { while } \xi_{2}>\frac{9}{4 \sqrt{6}}\left(1+\cos ^{2} \theta\right)\left(1-\cos ^{2} \theta\right)^{1 / 2}, \\
& \phi=2 \pi \xi_{3}
\end{aligned}
$$

which gives the expected distribution of samples; see Fig. 2(b).

To assess which rejection technique is the better, we should compare how tightly they each bound the Rayleigh phase

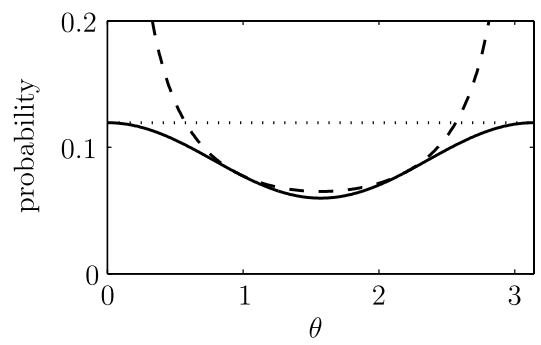

Fig. 3. Curves illustrating how tight the bound is for the different rejection sampling techniques. The solid curve is the Rayleigh phase function $p_{R}=\frac{1}{4 \pi} \frac{3}{4}\left(1+\cos ^{2} \theta\right)$, the dashed curve is $c$ pdf $=\frac{\sqrt{6}}{12}(1-$ $\left.\cos ^{2} \theta\right)^{-1 / 2}$, which is used for Baranoski's rejection sampling, and the dotted curve is $c \mathrm{pdf}=\frac{3}{2} \frac{1}{4 \pi}$, which is used when the unit sphere is sampled uniformly. 
function $p_{R}$ and we should compare them with respect to computational efficiency. Figure 3 illustrates that the uniform sampling of the sphere is the tighter bound. The pdf sampled with Baranoski's technique goes to infinity when the scattering angle goes to 0 or $\pi$. This means that a larger number of samples must be rejected in these areas. Baranoski's technique is also more computationally expensive as it requires that we call the cosine and square-root functions in the rejection sampling loop.

\section{B. Direct Sampling of Spherical Coordinates}

Another way to sample the Rayleigh phase function is to sample it directly. Direct sampling techniques are derived using the inversion method ([19], pp. 45-48). A four-step algorithmic description of the inversion method is provided by Pharr and Humphreys ([20], Subsection 14.3.1): (1) compute the cumulative distribution function $\operatorname{cdf}(x)$, (2) compute its inverse $\operatorname{cdf}^{-1}(x)$, (3) obtain a uniform, random number $\xi$, and (4) compute the sample value $X=\operatorname{cdf}^{-1}(\xi)$. This method is for sampling pdfs that take a one-dimensional argument. Since we would like to sample a direction on the unit sphere in spherical coordinates, we need to work with a twodimensional joint density function $p_{R}(\theta, \phi)=p_{R}(\cos \theta) \sin \theta$. We find a sampling technique for a joint density function by applying the inversion method in turn to the marginal density function $p_{R}(\theta)$ and the conditional density function $p_{R}(\phi \mid \theta)$ ([20], Subsection 14.5). These are

$$
\begin{aligned}
p_{R}(\theta) & =\int_{0}^{2 \pi} p_{R}(\theta, \phi) \mathrm{d} \phi \\
& =\int_{0}^{2 \pi} \frac{1}{4 \pi} \frac{3}{4}\left(1+\cos ^{2} \theta\right) \sin \theta \mathrm{d} \phi \\
& =\frac{3}{8}\left(1+\cos ^{2} \theta\right) \sin \theta \text { and } \\
p_{R}(\phi \mid \theta) & =\frac{p_{R}(\theta, \phi)}{p_{R}(\theta)}=\frac{1}{2 \pi} .
\end{aligned}
$$

The cumulative distribution function (cdf) for the conditional density function $p_{R}(\phi \mid \theta)$ is simply

$$
P_{R}(\phi \mid \theta)=\int_{0}^{\phi} \frac{1}{2 \pi} \mathrm{d} \phi^{\prime}=\frac{\phi}{2 \pi},
$$

and this is easily inverted to give $\phi=2 \pi \xi_{2}$. This leaves the marginal density function, which we will now find a sampling technique for using the inversion method. Deutschmann et al. [4] say that they use Cardan's formulas (also called Cardano's method [22]) for sampling the Rayleigh phase function, but they provide no further details. In the following, we shall see why Cardan's formulas are needed.

The cdf of the marginal density function $p_{R}(\theta)$ is

$$
\begin{aligned}
P_{R}(\theta) & =\int_{0}^{\theta} \frac{3}{8}\left(1+\cos ^{2} \theta^{\prime}\right) \sin \theta^{\prime} \mathrm{d} \theta^{\prime} \\
& ==-\frac{3}{8} \int_{1}^{\cos \theta}\left(1+x^{2}\right) \mathrm{d} x \\
& =-\frac{3}{8}\left[x+\frac{1}{3} x^{3}\right]_{1}^{\cos \theta} \\
& =\frac{1}{2}-\frac{3}{8} \cos \theta-\frac{1}{8} \cos ^{3} \theta
\end{aligned}
$$

This is a (depressed) cubic function with $x=\cos \theta$. To invert it, we need to find the root, and Cardan's formulas find the roots of cubic functions. A depressed cubic function is of the form

$$
x^{3}+a x+b=0 .
$$

Inserting $\xi_{1}$ in place of $P_{R}(\theta)$ and rewriting on this form, our equation has

$$
a=3, \quad b=4\left(2 \xi_{1}-1\right) .
$$

The solution is then, according to Cardan's formulas,

$$
x=\left[-\frac{b}{2}+\left(\frac{b^{2}}{4}+\frac{a^{3}}{27}\right)^{1 / 2}\right]^{1 / 3}+\left[-\frac{b}{2}-\left(\frac{b^{2}}{4}+\frac{a^{3}}{27}\right)^{1 / 2}\right]^{1 / 3},
$$

and this equation has only one real root if $\frac{b^{2}}{4}+\frac{a^{3}}{27}>0$. Inserting $a$ and $b$ from our equation, we can see that this condition is always fulfilled:

$$
\frac{b^{2}}{4}+\frac{a^{3}}{27}=\frac{16\left(2 \xi_{1}-1\right)^{2}}{4}+\frac{3^{3}}{27}=4(2 \xi-1)^{2}+1>0,
$$

since $\xi \in[0,1]$. This means that

$$
\begin{aligned}
\cos \theta= & {\left[-2\left(2 \xi_{1}-1\right)+\left(4\left(2 \xi_{1}-1\right)^{2}+1\right)^{1 / 2}\right]^{1 / 3} } \\
& +\left[-2\left(2 \xi_{1}-1\right)-\left(4\left(2 \xi_{1}-1\right)^{2}+1\right)^{1 / 2}\right]^{1 / 3}
\end{aligned}
$$

Looking at this equation, which samples $\cos \theta$ for the Rayleigh phase function directly, it seems quite computationally expensive as it apparently requires both a square root and two cube roots. Luckily, we can write Cardan's formula in a different way. Suppose we set

$$
u=\left[-\frac{b}{2}-\left(\frac{b^{2}}{4}+\frac{a^{3}}{27}\right)^{1 / 2}\right]^{1 / 3}
$$

then another way to write the solution is

$$
x=u-\frac{a}{3 u} \text {. }
$$

Or, if we insert the specific values for the Rayleigh phase function, the complete sampling technique is

$$
\begin{aligned}
u & =-\left[2\left(2 \xi_{1}-1\right)+\left(4\left(2 \xi_{1}-1\right)^{2}+1\right)^{1 / 2}\right]^{1 / 3}, \\
(\theta, \phi) & =\left(\cos ^{-1}(u-1 / u), 2 \pi \xi_{2}\right) .
\end{aligned}
$$

Figure 4 illustrates that both the simplified rejection technique and the direct sampling technique generate a similar even distribution of samples across the Rayleigh phase function shape.

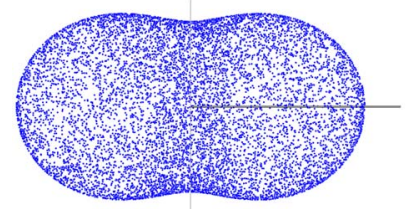

(a)

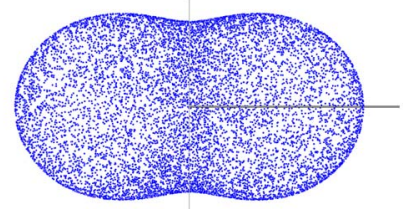

(b)
Fig. 4. (Color online) Spread of direction samples as points on the shape of the Rayleigh phase function. Directions sampled using (a) the simplified rejection technique and (b) the direct sampling technique, which employs Cardan's formulas. 


\section{Sampling by Tabulation}

There is one more sampling technique that is interesting to include in this investigation. When only measurements are available or when the analytical expression for a pdf is very complicated, it is common to tabulate the cdf and use this to sample the pdf as a step function. Sampling a step function using a tabulated cdf is fast. If the table has $n$ entries, the worst case complexity is $\log _{2} n$, as a lookup into the table is based on the binary search algorithm [23]. Even though the Rayleigh phase function is not measured nor complicated, it is interesting to see how this tabulation method performs as compared to the other techniques.

Suppose we pick $n$ samples $x_{i}, i=1, \ldots, n$, uniformly distributed across the domain of the pdf; then we can compute the $i$ th element in the table of cdf values by

$$
\operatorname{cdf}\left(x_{i}\right)=\sum_{j=1}^{i} \operatorname{pdf}\left(x_{j}\right) \Delta x=\operatorname{pdf}\left(x_{i}\right) \Delta x+\operatorname{cdf}\left(x_{i-1}\right),
$$

where $\Delta x$ is the distance between the samples and $\operatorname{cdf}\left(x_{0}\right)=0$. This is the general way of tabulating the cdf if we only have the pdf available. In the case of the Rayleigh phase function, we can tabulate the cdf of the marginal density function by

$$
\begin{aligned}
P_{R}\left(\cos \theta_{i}\right) & =P_{R}\left(x_{i}\right) \\
& =\sum_{j=1}^{i} p_{\mathrm{R}}\left(x_{j}\right) \Delta x \\
& =\frac{3}{8}\left(1+x_{i}^{2}\right) \Delta x+P_{\mathrm{R}}\left(x_{i-1}\right)
\end{aligned}
$$

with $\Delta x=2 / n$ and $x_{i}=1-i \Delta x$. However, the accumulation of values adds some numerical imprecision to the table. So, since we have a direct expression for $P_{R}$, a more exact tabulation is obtained by

$$
P_{R}\left(x_{i}\right)=\frac{1}{2}-\frac{3}{8} x_{i}-\frac{1}{8} x_{i}^{3}
$$

To sample the Rayleigh phase function using the tabulation of $P_{R}$, we need two random numbers $\left(\xi_{1}, \xi_{2}\right)$. One is used for a table lookup followed by linear interpolation to find $\cos \theta$; the other is used to find $\phi$ as in the sampling techniques described previously. The tabulation technique is then the following:

find $i$ such that $P_{R}\left(x_{i-1}\right) \leq \xi_{1}<P_{R}\left(x_{i}\right)$ using binary search ([르], Subsection 6.2.1),

$$
\begin{aligned}
\cos \theta & =1-\Delta x\left(i-\frac{P_{R}\left(x_{i}\right)-\xi_{1}}{P_{R}\left(x_{i}\right)-P_{R}\left(x_{i-1}\right)}\right) \quad \text { with } \\
P_{R}\left(\mathrm{x}_{0}\right) & =0, \quad \phi=2 \pi \xi_{2},
\end{aligned}
$$

and it gives the same results as the direct sampling technique [see Fig. 4(b)] except for the fact that the discretization introduces an error that depends on the number of table entries $n$. Each time we double the number of table entries, the error is quartered while one more iteration is required in the binary search.

\section{PERFORMANCE CONSIDERATIONS}

The number of samples that the different methods generate on average in equal time is provided in Table 1 . The simplified rejection technique performs better than the direct sampling. However, the samples seem to be slightly more clustered [compare Figs. 4(a) and 4(b)]. And the performance is slightly more unstable (as is often the case with rejection sampling). The tabulation method is the fastest method, but this comes at the cost of introducing error. However, since the Rayleigh phase function is not so complicated, we can get away with a table of relatively few entries. Using only 32 table entries, the root-mean-square error (rmse) is $2.9 \cdot 10^{-4}$, and the maximum error is $4.9 \cdot 10^{-4}$. Surprisingly, it is possible, as we shall see in the following, to optimize the simplified rejection sampling such that it performs better than the tabulation method unless we use a table with very few entries and accept a fairly large error.

A faster pseudorandom number generator improves the overall performance of all the methods, but it also improves the performance of the rejection methods more than it improves the direct sampling and the tabulation method. The $\mathrm{C}++$ standard library function rand was used for the performance test in Table 1 . Using a multiply-with-carry random number generator $[24,25]$ implemented in the NVIDIA OptiX framework [26,27], the average performance of the simplified rejection technique increases to 2.736 million samples given the same time for processing as in Table 1 . With the Mersenne Twister [28,29], the performance increases further to 2.990 million samples. The tabulation method cannot benefit as much from a faster random number generator. For comparison, its average performance increases to 2.920 million samples with the Mersenne Twister and $n=32$ table entries. To beat the simplified rejection sampling using tabulation, we have to decrease the number of table entries to $n=8$. Then we get 3.080 million samples, but the rmse increases to $4.5 \cdot 10^{-3}$, which is nearly the expected 16 -fold increase. When using a fast random number generator, the performance

Table 1. Number of Samples in Millions Generated by the Different Importance Sampling Methods in Equal Time (1 s on a Laptop Computer)

\begin{tabular}{lcccccc}
\hline Trial No. & von Neumann & Spherical Coordinates & Baranoski & Simplified Rejection & Direct & Tabulation $n=32$ \\
\hline 1 & 1.603 & 2.207 & 2.171 & 2.477 & 2.204 & 2.738 \\
2 & 1.628 & 2.207 & 2.247 & 2.513 & 2.263 & 2.660 \\
3 & 1.592 & 2.195 & 2.225 & 2.487 & 2.272 & 2.749 \\
4 & 1.633 & 2.199 & 1.916 & 2.472 & 2.264 & 2.671 \\
5 & 1.651 & 2.219 & 2.210 & 2.416 & 2.249 & 2.735 \\
Average & 1.621 & 2.205 & 2.154 & 2.473 & 2.710 \\
\hline
\end{tabular}


gain that we can get from using the tabulation method hardly justifies the error.

In the performance test, the implementation of the direct sampling uses the $\mathrm{C}++$ standard library function pow to find the needed cube root. This is an expensive function to call. If we implement a function specifically for finding cube roots, we can obtain better performance while retaining the same level of precision. Using Kahan's method for finding a quick approximation to the cube root [30] and Lancaster's iteration formula to obtain the desired precision [31] (two iterations suffice), the average performance of the direct sampling method increases to 2.606 million samples given the same time for processing as in Table $\underline{1}$ and using the Mersenne Twister for random number generation. Code for computing a cube root in this way as well as performance and precision tests have been posted online by a blogger [32]. It is interesting to note that, with the standard rand as random number generator and the improved function for finding cube roots, the direct sampling has performance much closer to the simplified rejection sampling (2.406 million samples on average). And it actually outperforms the simplified rejection sampling if we accept lower precision in the cube-root function (using just one iteration with Lancaster's formula gives 2.540 million samples on average). With the Mersenne Twister, the simplified rejection sampling seems unbeatable.

In conclusion, the simplified rejection sampling technique, which samples the cosine of the scattering angle by $2 \xi_{1}-1$, is the most efficient way of sampling the Rayleigh phase function. This is true unless we use an expensive random number generator. If we choose an expensive random number generator, the tabulation method is the fastest as long as we can accept a small error. Otherwise, the direct sampling technique is useful, but its performance depends on the efficiency of the cube-root function used. In comparison to simplified rejection sampling, tabulation and direct sampling have slightly more stable performance, and the samples are perhaps slightly less clustered.

\section{REFERENCES AND NOTES}

1. R. J. D. Tilley, Colour and the Optical Properties of Materials, 2nd ed. (Wiley, 2011).

2. G. N. Plass and G. W. Kattawar, "Radiative transfer in an atmosphere-ocean system," Appl. Opt. 8, 455-466 (1969).

3. E. J. McCartney, Optics of the Atmosphere: Scattering by Molecules and Particles (Wiley, 1976).

4. T. Deutschmann, S. Beirle, U. Friess, M. Grzegorski, C. Kern, L. Kritten, U. Platt, C. Prados-Román, J. Puḳ̄te, T. Wagner, B. Werner, and K. Pfeilsticker, "The Monte Carlo atmospheric radiative transfer model McArtim: introduction and validation of Jacobians and 3D features," J. Quant. Spectrosc. Radiat. Transfer 112, 1119-1137 (2011).

5. A. Kienle, F. K. Forster, and R. Hibst, "Influence of the phase function on determination of the optical properties of biological tissue by spatially resolved reflectance," Opt. Lett. 26, 1571-1573 (2001).

6. A. Krishnaswamy and G. V. G. Baranoski, "A study on skin optics," Tech. Rep. CS-2004-01 (University of Waterloo, 2004).
7. M. W. Y. Lam and G. V. G. Baranoski, "A predictive light transport model for the human iris," Comput. Graph. Forum 25, 359-368 (2006).

8. G. V. G. Baranoski and A. Krishnaswamy, "Light interaction with human skin: from believable images to predictable models," SIGGRAPH Asia 2008, Course Notes (2008).

9. G. V. G. Baranoski and A. Krishnaswamy, Light \& Skin Interactions: Simulations for Computer Graphics Applications (Morgan Kaufmann/Elsevier, 2010).

10. B. W. Whitney and L. Hartmann, "Model scattering envelopes of young stellar objects. I. Method and application to circumstellar disks," Astrophys. J. 395, 529-539 (1992).

11. S. Seager, B. A. Whitney, and D. D. Sasselov, "Photometric light curves and polarization of close-in extrasolar giant planets," Astrophys. J. 540, 504-520 (2000).

12. S. Lallich, F. Enguehard, and D. Baillis, "Radiative properties of silica nanoporous matrices," Int. J. Thermophys. 29, 1395-1407 (2008).

13. L. G. Henyey and J. L. Greenstein, "Diffuse radiation in the galaxy," Ann. Astrophys. 3, 117-137 (1940). Also in Astrophys. J. 93, 70-83 (1941).

14. W. M. Cornette and J. G. Shanks, "Physically reasonable analytical expression for the single-scattering phase function," Appl. Opt. 31, 3152-3160 (1992).

15. Q. Liu and F. Weng, "Combined Henyey-Greenstein and Rayleigh phase function,” Appl. Opt. 45, 7475-7479 (2006).

16. J. W. Strutt, "On the light from the sky, its polarization and colour," Philos. Mag. 41, 107-120, 274-279 (1871). Reprinted in John William Strutt (Baron Rayleigh), Scientific Papers (Cambridge University, 1899), Vol. 1, No. 8, pp. 87-103.

17. S. Chandrasekhar, Radiative Transfer (Oxford, 1950).

18. J. von Neumann, "Various techniques used in connection with random digits," in Monte Carlo Method, A. S. Householder, ed., Vol. 12 of Applied Mathematics Series (National Bureau of Standards, 1951), Chap. 13, pp. 36-38.

19. M. H. Kalos and P. A. Whitlock, Monte Carlo Methods (Wiley, 1986), Vol. 1.

20. M. Pharr and G. Humphreys, Physically Based Rendering: From Theory to Implementation (Morgan Kaufmann/Elsevier, 2004).

21. J. F. Hughes and T. Möller, "Building an orthonormal basis from a unit vector," J. Graphics Tools 4, 33-35 (1999).

22. "Cubic function," Wikipedia, http://en.wikipedia.org/wiki/ Cubic_function.

23. D. E. Knuth, The Art of Computer Programming, 2nd ed. (Addison-Wesley, 1998), Vol. 3.

24. G. Marsaglia, "Yet another RNG" (1994). Posted to the Usenet group sci.stat.math.

25. G. Marsaglia, "Random number generators," J. Mod. Appl. Stat. Meth. 2, 2-13 (2003).

26. S. G. Parker, J. Bigler, A. Dietrich, H. Friedrich, J. Hoberock, D. Luebke, D. McAllister, M. McGuire, K. Morley, A. Robison, and M. Stich, "OptiX: a general purpose ray tracing engine," ACM Trans. Graph. 29, 1-13 (2010).

27. "NVIDIA OptiX 2 ray tracing engine," http://developer.nvidia .com/optix.

28. M. Matsumoto and T. Nishimaru, "Mersenne Twister: a 623dimensionally equidistributed uniform pseudorandom number generator," ACM Trans. Model. Comput. Simul. 8, 3-30 (1998).

29. "Mersenne Twister," http://www.math.sci.hiroshima-u.ac.jp/ $\sim$ m-mat/MT/emt.html.

30. W. Kahan, "Computing a real cube root," lecture notes (1991), http://www.cims.nyu.edu/ dbindel/class/cs279/qbrt.pdf. Retypeset by D. Bindel, April 2001.

31. O. E. Lancaster, "Machine method for the extraction of cube root," J. Am. Stat. Assoc. 37, 112-115 (1942).

32. Metamerist, "In search of a fast cube root," http://metamerist .com/cbrt/cbrt.htm. 